\title{
First measurements and observations from the new permanent magnet undulator at the SRC
}

\author{
D. J. Wallace, ${ }^{\text {a) }}$ J. J. Bisognano, M. Bissen, D. Eisert, M. A. Green, T. Kubala, \\ G. C. Rogers, and W. S. Trzeciak \\ SRC, UW-Madison, 3731 Schneider Drive, Stoughton, Wisconsin 53589
}

(Presented on 22 August 2001)

\begin{abstract}
In December of 2000 the University of Wisconsin Synchrotron Radiation Center (SRC) installed a new, state-of-the-art undulator in long straight section 2 of the Aladdin storage ring. This undulator replaced the first Aladdin undulator, which was on loan from the Stanford Synchrotron Radiation Laboratory and had been in use since 1986 [K. Halbach et al., IEEE Trans. Nucl. Sci. NS-28, 3136 (1981); H. Winick et al., Nucl. Instrum. Methods Phys. Res. A 208, 127 (1983); M. A. Green et al., Nucl. Instrum. Methods Phys. Res. A 266, 91 (1988)]. The new undulator was designed, constructed, and tested by Danfysik (Danfysik A/S, Moellchaves 31A, DK-4040 Jyllinge, Denmark). The undulator uses $\mathrm{NdFeB}$ permanent magnets for the magnetic structure and is $3.52 \mathrm{~m}$ long with 50 periods, each of $68.3 \mathrm{~mm}$. The new undulator is intended to provide high flux from 7.8 to $500 \mathrm{eV}$. To cover this wide energy range we use the first through ninth odd harmonics. The magnetic structure has been phase-corrected to better than $2^{\circ} \mathrm{rms}$ and should provide fidelity better than $90 \%$ through the ninth harmonic. The resulting undulator flux will support an extreme ultraviolet nano-lithography beamline, a low energy Wadsworth beam line, and a high energy VLS-PGM beam line. Some coherence experiments are also anticipated. The undulator radiation has been analyzed using an existing HTM [M. C. Hettrick, Nucl. Instrum. Methods Phys. Res. A 266, 404 (1988)] monochromator, a durable filter-pinhole detector, and computer controlled, cooled, scanning apertures. We present measurements of the undulator harmonic content, energy and spatial structure of the undulator beam, and undulator beam position stability. (c) 2002 American Institute of Physics. [DOI: 10.1063/1.1445487]
\end{abstract}

\section{INTRODUCTION}

A new state-of-the-art pure permanent magnet undulator (U2) has been installed in long straight section 2 of the Aladdin storage ring. This undulator was designed and constructed with very tight magnetic and mechanical specifications to provide high flux and spectral purity for the first through ninth odd harmonics. This will allow proper functionality over the wide intended energy range from 7.8 to $\sim 500 \mathrm{eV}$ (see Table I). The U2 was subjected to rigorous magnetic and mechanical testing before installation and alignment on the storage ring.

The undulator presently provides photons to an extreme ultraviolet (EUV) lithography exposure station, operated by CNTech, and a high-throughput monochromator (HTM) which had been used with the previous undulator. The HTM was left in place to serve as a diagnostic tool for U2 evaluation. To better utilize the high U2 flux, a $5 \mathrm{~m}$ Wadsworth beamline is being constructed to provide high resolution photons from 7.8 to $50 \mathrm{eV}$ with a small final image size. This beamline is optimized for the study of magnetic materials using spin-polarized photoemission. A high flux VLS-PGM beamline will also provide photons for carbon, nitrogen, and possibly oxygen spectroscopy.

${ }^{a)}$ Electronic mail: danwallace@src.wisc.edu

\section{MECHANICAL DESIGN AND CONTROL}

The U2 pole magnets are attached to two stainless steel girders that are each supported by two lead screws which are connected to a steel " $\mathrm{C}$ " frame. The drive system has two identical assemblies located $0.96 \mathrm{~m}$ upstream and downstream of the undulator centerline. Both assemblies consist of a stepping motor, gear reducer, clutch, brake, and lead screw. This dual drive enables correction of longitudinal taper. There is also an inclinometer located on the lower girder to ensure that the girders remain level.

The undulator scans from a minimum gap of $23.82 \mathrm{~mm}$ to a maximum gap of $200 \mathrm{~mm}$ with a resolution of $0.2 \mu \mathrm{m}$ and a repeatability of $3.0 \mu \mathrm{m}$. Four Heidenhain linear encoders, between the four corners of the two girders, enable correction of gap errors from girder roll that is necessary for the repeatability and control of the higher harmonic energies versus gap. Sixteen limit switches protect the undulator mechanism from overtravel. The large number of limit switches enables the undulator to have a warning and disable limit at each end of the two girders, for both opening and closing. The limit switches and inclinometer are monitored by a programmable logic controller (PLC) that in turn supplies status signals to the control computer and enable signals for the motors and brakes. The computer contains control modules for the stepping motors, reading the status signals and the linear encoder signals. The control software employs closed 
TABLE I. U2 design parameters.

\begin{tabular}{ll}
\hline \hline Type of magnet & Pure, permanent magnets \\
\hline Pitch, $\lambda_{U}$ & $68.3 \mathrm{~mm}$ \\
Overall length & $3.52 \mathrm{~m}$ \\
Pole width & $94 \mathrm{~mm}$ \\
Number of full-strength poles & 101 \\
Maximum field and $K$ value & $7.17 \mathrm{kG}, 4.56$ \\
Minimum magnetic gap & $23.82 \mathrm{~mm}$ \\
Useful harmonics & $1 \mathrm{st}, 3 \mathrm{rd}, 5 \mathrm{th}, 7 \mathrm{th}, 9 \mathrm{th}$ \\
\hline \hline
\end{tabular}

loop feedback to adjust the gap opening using the linear encoders and stepping motors.

\section{MAGNETIC PERFORMANCE}

Proper undulator operation requires high magnetic field quality and mechanical integrity in order to produce photons with the proper harmonic content and not adversely affect the storage ring operation. Contractual requirements with Danfysik included complete retesting, and any necessary corrections of the undulator onsite, after shipment from Denmark. Notable in the specifications were achieving a magnetic field strength capable of placing the first-harmonic peak at or below $7.8 \mathrm{eV}$ at $800 \mathrm{MeV}$ ring energy; the ability to produce ninth-harmonic radiation at least $90 \%$ of theoretical; and multipole field quality, with tight limitation, which would ensure adequate stability of the electron beam in the storage ring. Magnetic testing utilized both a scanning Hall probe referenced to a 6-m precision granite block and also full-length untwisted (first-integral) and twisted (secondintegral) integration coils.

Confirming field strength at minimum gap to achieve the $7.8 \mathrm{eV}$ was straightforward. Maintaining $90 \%$ ninthharmonic fidelity was translated into a $2^{\circ} \mathrm{rms}$ phase error requirement which was easily assessed from the Hall probe measurements. Field measurements indicated the necessity of a special saddle-coil to finely correct a subtle, and unexplained, long-wave field error. Trim coils included long- $B_{x}$, long- $B_{y}$, short- $B_{y}$ at the ends, and the saddle-coil. The long$B_{y}$ coils were electrically separated, with two supplies, which can be mixed for horizontal steering and/or skew quad correction. No skew quad correction was necessary to meet the specifications. All trim coils were calibrated and optimal excitations were determined versus gap. Three alignment targets located on the upper girder and three leveling plates were referenced to the magnetic center planes of the undulator as determined by these field measurements.

Subsequent commissioning lead to the following operation. ${ }^{1}$ Closed orbit steering control is achieved with a combination of the internal undulator trims, fed-forward local steerers, and the global orbit feedback system. Focusing corrections include lookup-table local quadrupole shunts and global tune control that is both fed-back and fed-forward. Typical electron beam stability requirements are, vertically, $\delta y \leqslant 10 \mu \mathrm{m}$ and $\left|\delta \sigma_{y} / \sigma_{y}\right| \leqslant 1.5 \%$ for size, and no adverse variations in lifetime. Attaining these goals is presently understood and will be fully achieved with implementation of the new low-emittance lattice, ${ }^{2}$ new optical monitoring stations, and better control of coupling effects.

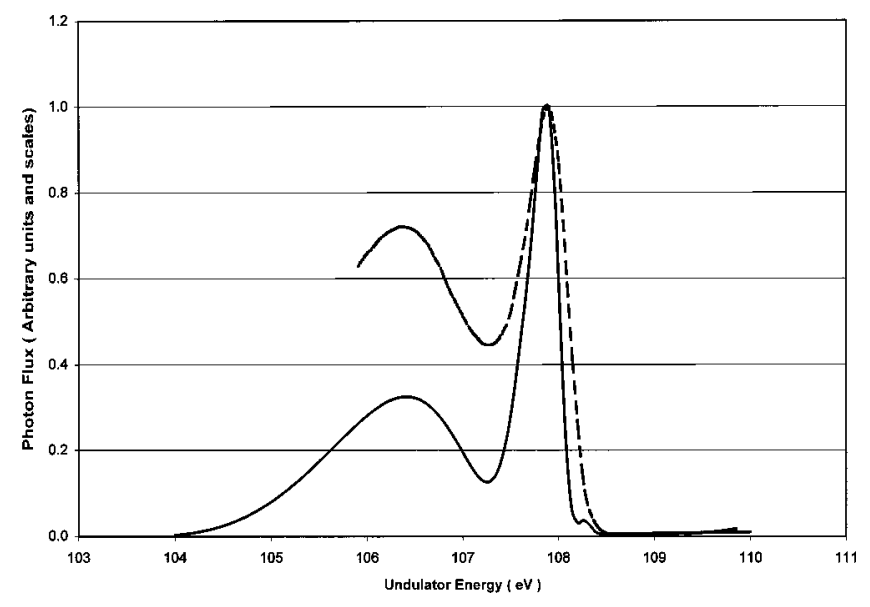

FIG. 1. U2 ninth harmonic peak as measured (dashed line) and as calculated (solid line). Measurement details: $12 \mathrm{eV}$ fundamental energy, $3.585 \mathrm{~K}$ value, $800 \mathrm{MeV}$ ring energy, and $1 \times 1 \mathrm{~mm}^{2}$ cooled apertures. Peak width for measured harmonic is $0.44 \mathrm{eV}$ compared to a total theoretical peak width of 0.43 $\mathrm{eV}$. Vertical scale is linear and adjusted for peak comparison.

\section{MEASUREMENTS OF SPECTRAL PERFORMANCE}

Using the existing HTM monochromator as a diagnostic tool, medium resolution scans were made for fundamental and harmonic peaks within the 40-150 eV HTM energy range. Reasonable agreement was found between the measured widths of the $\mathrm{U} 2$ peaks and peaks from modeling with XOP/URGENT ${ }^{3}$ computations.

Since the U2 is expected to cover a wide energy range using the first through ninth odd harmonics, it was critical to confirm the spectral quality of the ninth harmonic. With the $\mathrm{U} 2$ gap set for a fundamental of $12 \mathrm{eV}$ and $1 \times 1 \mathrm{~mm}^{2}$ cooled apertures, the measured peak width of the ninth harmonic (at $108 \mathrm{eV}$ ) was determined to be $0.44 \mathrm{eV}$. The principle contributions to this measured width are the ideal peak width as calculated by XOP/URGENT of $0.26 \mathrm{eV}$, the electron energy spread of $0.23 \mathrm{eV}$, and the broadening due to monochromator resolution of $0.25 \mathrm{eV}$. When these values are added in quadrature the result is $0.43 \mathrm{eV}$ which compares favorably with the measured value of $0.44 \mathrm{eV}$ (see Fig. 1). It should be noted that the skewed shape of the measured peak results from the monochromator transfer function.

Of particular importance is the low energy limit of the U2 output radiation. CNTech and other semiconductor research facilities will be using $157 \mathrm{~nm}(7.8 \mathrm{eV})$ for the next generation of UV photolithography. Future photoresists, effective exposure techniques, and reflective image formation optics are being studied in this wavelength region. The U2 specification required photon production at $7.8 \mathrm{eV}$ to support these studies. Since the energy range of the HTM is 40-150 $\mathrm{eV}$, effective scanning of the $7.8 \mathrm{eV}$ fundamental peak was not directly possible. A system was devised to use the HTM optics at zero order as a collector to feed a $0.3 \mathrm{~m}$ McPherson scanning monochromator. This system had many losses and low resolution but was sufficient to establish the low energy U2 limit at about $7.94 \mathrm{eV}$ (see Fig. 2). This difference from the specification is most likely from a storage ring energy that is $\sim 1 \%$ higher than expected and is consistent with results from the U1 and U3 undulators. 


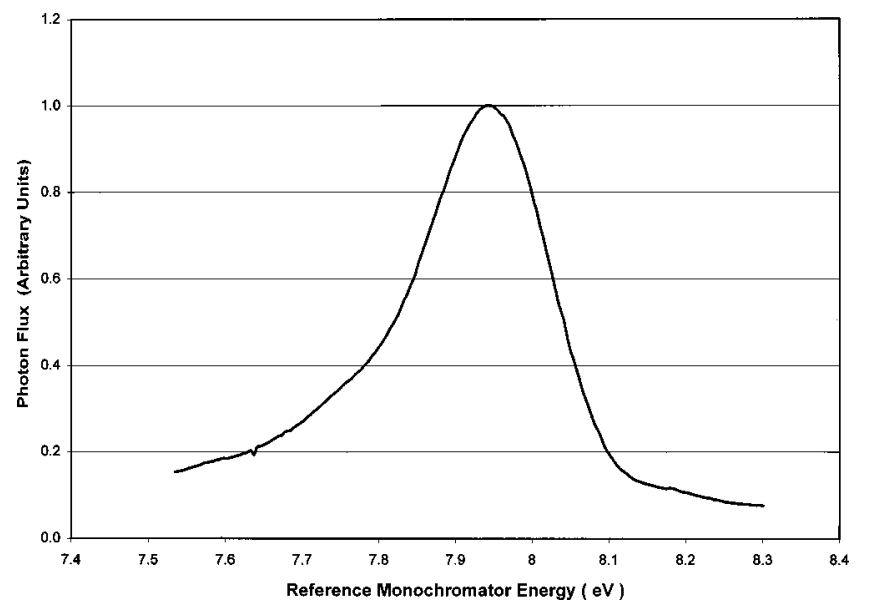

FIG. 2. Lowest energy output measured from U2. Measurement details: gap closed to $23.823 \mathrm{~mm}$, ring energy $\sim 800 \mathrm{MeV}$, and cooled apertures at 1 $\times 1 \mathrm{~mm}^{2}$. Measured with HTM at zero order and a $0.3 \mathrm{~m} \mathrm{McPherson}$ as the reference monochromator.

\section{MEASUREMENTS OF SPATIAL STRUCTURE}

Two additional diagnostic tools were available to analyze the U2 radiation. A scanning pinhole detector and cooled scanning apertures provided data on the spatial (and energy) structure of the photon beam. The scanning pinhole detector used for these $\mathrm{U} 2$ measurements is an upgraded version of the system ${ }^{4}$ used on the U1 beamline and the original U2 beamline for alignment and testing. The detector consists of a heat sink shield, a $500 \mu \mathrm{m}$ pinhole, and an XUV-100-G $\mathrm{G}^{5}$ silicon photodiode coated with a $139 \mathrm{~nm}$ thick aluminum filter (see Fig. 3). The aluminum filter is intended to decrease the U2 flux by a factor of $\sim 4$ and to provide some energy discrimination in the scans by using the aluminum $L_{23}$ edge at $73 \mathrm{eV}$. Previous scanning pinhole measurements were limited to ring currents less than $10 \mathrm{~mA}$, due primarily to thermal damage to the thin filters. The combination of the silicon photodiode bulk detector and the evaporated aluminum filter allowed measurements at ring currents closer to actual ring operation. For an undulator energy of $73 \mathrm{eV}$, the detector was stable and linear to $\sim 1 \%$ for ring currents up to $40 \mathrm{~mA}$ at $800 \mathrm{MeV}$. This U2 energy provides a useful signal dip from the detector at the $\mathrm{U} 2$ beam center due to the aluminum filter edge and the undulator beam energy structure (see Fig. 4, scan No. 1). For higher ring currents and/or lower photon energies with more flux, detector saturation occurred (see Fig. 4, scans No. 3 and No. 4) and for the highest undulator fluxes an $8 \%$ nonrecoverable degradation occurred. At $73 \mathrm{eV}$ the aluminum filter survived ring currents as high as 135 $\mathrm{mA}$.

Extensive vertical scans were taken with the pinhole detector located $9.3 \mathrm{~m}$ from the U2 source. Vertically the detector was reproducible to better than $20 \mu \mathrm{m}$ at a U2 energy of $73 \mathrm{eV}$. Day-to-day vertical beam repeatability was better than $60 \mu \mathrm{m}$. Over a U2 scan range from 73 to $7.8 \mathrm{eV}$ (gap 65.2 to $23.8 \mathrm{~mm}$ ) the beam was stable to better than $50 \mu \mathrm{m}$. These changes in vertical position are likely due to a combination of experimental error, small angular changes in beam pointing $(5 \mu \mathrm{rad})$, detector mechanical stability, and probable thermal elongation.

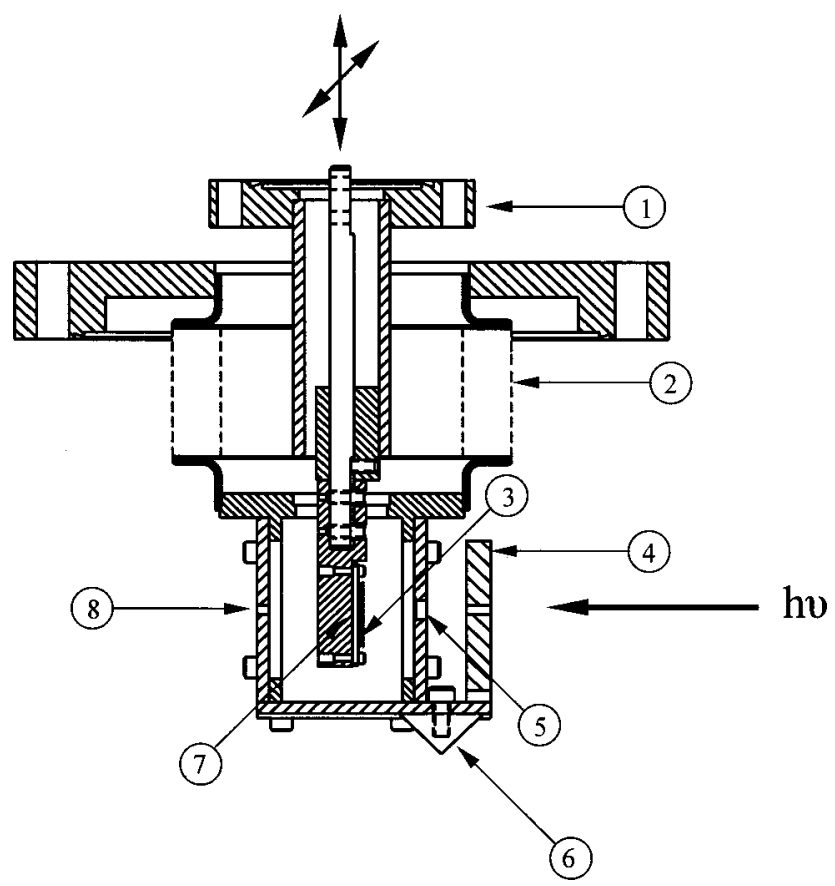

FIG. 3. Details of scanning pinhole for undulator beam analysis. Components: 1: horizontal and vertical scanning stage; 2 : bellows for horizontal and vertical motion; 3: $1390 \AA$ aluminum filter; 4: heat sink shield; 5: 500 $\mu \mathrm{m}$ pinhole; 6: prism for beam tracking with laser; 7: XUV-100-G silicon photodiode detector (IRD Inc.); and 8: alignment access hole. Note: additional vacuum and mechanical components are removed for clarity.

The U2 cooled apertures are constructed of massive oxygen-free high-conductivity copper blades with internal water cooling. They are computer controlled for accurate motions in the horizontal and vertical directions. The aperture scanning program allows rastering of a small aperture across the incident U2 beam with the HTM passing a particular energy and bandwidth. In this way a spatial map can

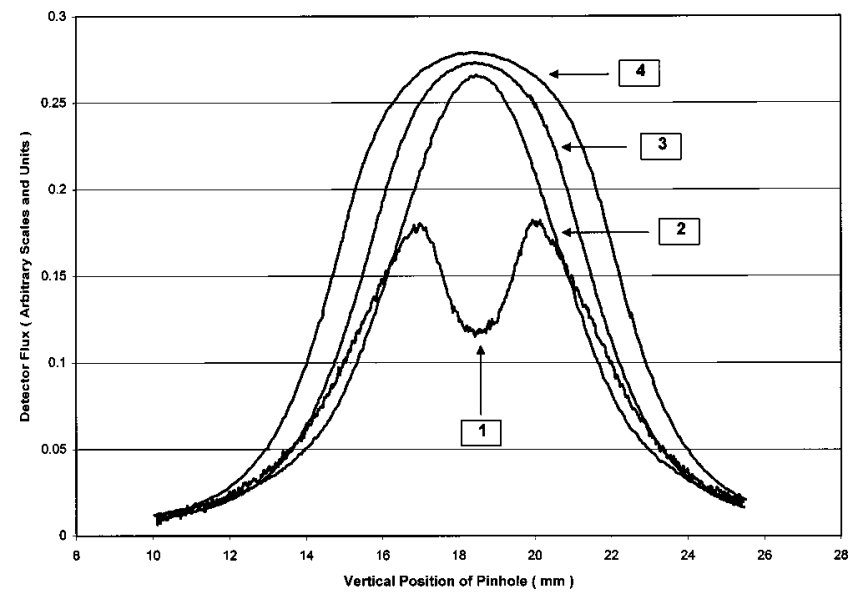

FIG. 4. Pinhole detector scans showing repeatability of undulator vertical beam position for four different undulator gaps. Scans have bending magnet background flux subtracted out. Scan (1) shows the $73 \mathrm{eV}$ aluminum absorption dip. Scans (3) and (4) clearly show saturation due to higher fluxes at lower energies. Details: scan (1) gap was $65.2 \mathrm{~mm}$ with fit center at 18.492 $\mathrm{mm}$, scan (2) gap was $36 \mathrm{~mm}$ with fit center at $18.487 \mathrm{~mm}$, scan (3) gap was $28.4 \mathrm{~mm}$ with fit center at $18.469 \mathrm{~mm}$, and scan (4) gap was $23.8 \mathrm{~mm}$ with fit center at $18.448 \mathrm{~mm}$. Vertical scales are adjusted for clarity. 
be made of the undulator beam for a given energy. These results seem reasonable and are presently being analyzed.

\section{FIRST USER EXPERIMENTS}

During the U2 testing CNTech proceeded with EUV lithographic exposures. In addition to standard exposures, successful results were achieved with Lloyds mirror and grating interferometric methods using the HTM and the U2 third harmonic at $\sim 92 \mathrm{eV}$. These preliminary exposures indicate a reasonable photon beam coherence. (These results will be reported by $\mathrm{CNTech}$ at a later date.) $\mathrm{CNTech}$ reported that the higher flux from the new U2 has decreased exposure times by a factor of 10 . While the HTM beamline was configured for measuring $157 \mathrm{~nm}$ photons, successful exposures were also made with the photo resist UV6.

\section{CONCLUSION}

While there is much more to investigate and analyze, it is clear that the $\mathrm{U} 2$ produces a ninth harmonic of appropriate width and produces significantly increased fluxes. The positional stability of the U2 beam is acceptable over a wide energy-gap range. These results with actual undulator radiation attest to the validity of the magnetic testing and mechanical design.

\section{ACKNOWLEDGMENTS}

The authors wish to thank the CNTech staff for their cooperation during the initial conditioning and testing phase of the new U2 at the SRC. This work is based upon research conducted at the Synchrotron Radiation Center, University of Wisconsin-Madison, which is supported by the NSF under Award No. DMR-0084402.

\footnotetext{
${ }^{1}$ M. A. Green et al., Proceedings of the Particle Accelerator Conference, Chicago, IL, 2001.

${ }^{2}$ J. J. Bisognano et al., Proceedings of the Particle Accelerator Conference, Chicago, IL, 2001.

${ }^{3}$ M. Sanchez del Rio and R. J. Dejus, Proc. SPIE 2448, 340 (1998).

${ }^{4}$ M. A. Green et al., Nucl. Instrum. Methods Phys. Res. A 347, 568 (1994).

${ }^{5}$ International Radiation Detectors, Inc., Torrence, CA.
} 